\title{
US preexposure: Effects on flavor aversions produced by pairing a poisoned partner with ingestion
}

\author{
SAM REVUSKY, SHANNON COOMBES, and RICHARD W. POHL \\ Memorial University, St. John's, Newfoundland A1B 3X9, Canada
}

\begin{abstract}
If an unpoisoned rat drinks a flavored solution and then is exposed to another rat suffering from lithium poisoning, the unpoisoned rat will later exhibit a flavor aversion. This is called the poisoned partner effect (PPE). The present experiments showed that (1) the US preexposure effect applies to the PPE since, if the unpoisoned rat is preexposed to poisoned partners, the PPE is attenuated, (2) the magnitude of the PPE increases with the dose of poison, and (3) a poisoned partner accustomed to poisoning produces a slightly weaker PPE than a partner naive to poisoning. Two earlier findings were confirmed: (1) The PPE occurs because the poisoned partner is an aversive US, not because a learned aversion is transferred from one rat to another; and (2) the PPE is weaker than an aversion produced by poisoning itself.
\end{abstract}

An unpoisoned rat that drinks saccharin solution and is subjected to the presence of a poisoned rat in its home cage as much as $6 \mathrm{~h}$ later will develop a saccharin aversion (Coombes, Revusky, \& Lett, 1980; Lavin, Freise, \& Coombes, 1980). The poisoned rat is called a poisoned partner (PP) and the aversion exhibited by the unpoisoned subject rat is called the PP effect (PPE).

\section{EXPERIMENT 1}

Experiment 1 was concerned with the possibility that preexposure to PPs may have the same detrimental effects on learning as does preexposure to other USs (Braveman, 1975; Cappell \& LeBlanc, 1977; Gamzu, 1977; Mis \& Moore, 1973; Rescorla, 1973; Revusky \& Taukulis, 1975). The critical pair of treatments involved first exposing some rats to the presence of PPs and exposing other rats to the presence of unpoisoned partners (UPs). Then, in both treatments, the rats were conditioned by being ailowed to drink saccharin solution prior to being exposed to the presence of a PP. A US preexposure effect would be obtained if higher preferences for saccharin (and hence weaker learned aversions) were obtained among the rats preexposed to the PP.

Although the main point involved the comparison of these two pretreatments, to use our resources (in-

Supported by the National Research Council of Canada. We thank Bow Tong Lett and Gerard M. Martin for critically reading the manuscript. We would like to take this opportunity to thank Michael Lavin for discovering the poisoned partner effect, which has afforded us much amusement. For the honest apportionment of scientific credit, we state that none of the present authors were in on the original discovery. cluding the PPs) efficiently, we also dealt with a number of secondary issues. The procedure outlined in the preceding paragraph results in some partners' being preexposed to the toxin and others not. Since it was desirable to use both sets of partners as PPs during the conditioning phase, we factored out the effect of preexposure of the PP to the toxin. We guessed that such preexposure might weaken the PPE by reducing the aversiveness of the PPs. We do not understand what underlies the PPE and therefore had no rigorous basis for this guess. Still, it seemed likely that the sickness produced by the toxic agent would be lessened if the PP were preexposed to it. This preexposure presumably would weaken the intensity of the unknown US, produced by the PP, that is responsible for the PPE. If so, the PPE would be weakened by the US decrement resulting from familiarity of the PP with sickness. We also added a third factor. This was whether the PP was allowed to develop an aversion to saccharin solution on the day of conditioning by being administered saccharin solution prior to being placed with the subject rat. Coombes et al. (1980) found that this factor did not affect the magnitude of the PPE. If so, the PPE does not depend on the development of a saccharin aversion in the PP, and hence the PPE cannot be considered transfer of a learned aversion from the PP to the subject rat.

The three factors described in the preceding paragraph were combined in a $2 \times 2 \times 2$ factorial design (eight groups): (1) whether there were preexposures to PPs or to UPs; (2) whether the PP was familiar with the poison on the conditioning day; and (3) whether the PP consumed saccharin solution prior to being used as a US on the conditioning day. To present the data and define groups, we pooled the 
last factor since, as expected, it had no effect. Thus, four groups of 10 rats each were defined on the basis of the first two factors. The groups were named with a dual nomenclature that indicated first the type of preexposure, to PPs or to UPs, and then, after a slash, what happened during conditioning. For instance, Group PP/Sac $\rightarrow$ nPP means the subject rat was preexposed to PPs; during conditioning, it first drank saccharin solution alone and then was paired with a PP for which the poisoning was novel. Group $\mathrm{PP} / \mathrm{Sac} \rightarrow \mathrm{fPP}$ refers to preexposure to PPs and conditioning in which saccharin solution was consumed prior to pairing with a PP for which poisoning was familiar. Group UP/Sac $\rightarrow$ nPP refers to preexposure to UPs and conditioning with nPPs. Group UP/Sac $\rightarrow$ fPP refers to preexposure to UPs and conditioning with fPPs.

A learned flavor aversion was presumed to have developed in one of the preceding groups if its preference for saccharin during testing was lower than that of Group Cont, which was an analog of the traditional unpaired CS-US control group. In the flavor aversions usually studied, the USs are strong toxins that produce an obvious decline in preference from the day of conditioning to the day of testing. In the case of weak aversions, such as those produced by PPs, there is often no such obvious decline, and there even may be an increase in flavor preference from training to testing. The reason is that the learned aversion is counteracted by a decline in neophobia to the novel flavor resulting from the exposure to it on the day of conditioning (Revusky, 1978, 1979). In the case of a very weak aversion, this increase in preference due to habituation may be greater than the decrease in preference due to the aversion (Revusky, Taukulis, Parker, \& Coombes, 1979). The role of neophobia was particularly prominent in the present experiments due to the use of a high concentration of saccharin solution, which increases neophobia but was nevertheless desirable because it also enhances conditioning. Thus, it was necessary to define the aversions in the PPE groups in terms of a test preference that was lower than Group Cont's instead of in terms of a decrease in preference from the day of conditioning to the day of testing. We do not think there is any reason to attribute different learning mechanisms (e.g., interference with habituation to a novel taste) to such weak aversions and to strong aversions resulting in a marked decline in flavor preference, although the point might be debated.

Subgroups within Group Cont were subjected to different procedures. Some were treated like the rats in the $2 \times 2 \times 2$ factorial design except that they did not drink saccharin solution before being paired with a PP on the conditioning day. Others consisted of rats that functioned as PPs on the conditioning day, but did not drink saccharin solution prior to being injected with the lithium toxin. The pooling of un- paired CS-US control groups with USs very different in strength was based on earlier findings that if sickness unpaired with drinking has dissipated when the animal is exposed to a flavored solution, there is no nonassociative effect of the US on saccharin preference (Carroll, Dinc, Levy, \& Smith, 1975; Domjan, 1977; Revusky, Parker, Commbes, \& Coombes, 1976). Of course, this assumption was subject to statistical confirmation.

The remaining two groups consisted of the PPs that drank saccharin solution before being poisoned on the day of conditioning. Group $\mathrm{Sac} \rightarrow \mathrm{fLi}$ was familiar with lithium sickness at the time of conditioning, while Group Sac $\rightarrow \mathrm{nLi}$ was not. These two groups allowed assessment of the effects of preexposure to lithium sickness on flavor aversion conditioning among the PPs and a comparison of the PPEs with aversions produced directly by poisoning.

\section{Method}

The 96 male Sprague-Dawley rats ranged in ad-lib weight from 197 to $228 \mathrm{~g}$ just before the experiment began. They had been ordered from Canadian Breeding Laboratories in terms of body weight, but were probably 7 or 8 weeks old. All procedures were conducted in stainless steel mesh $(1.27-\mathrm{cm}$ grids) home cages. The rats were deprived of water on the day before the experiment began (Day 0 ), and fluids were available oniy when specifically indicated. Dry Purina Chow was available throughout.

Forty rats were subjected to the $2 \times 2 \times 2$ set of treatments (five rats per cell) that fit the paradigm which has produced PPEs; that is, during conditioning, they were not poisoned but drank saccharin solution prior to being exposed to PPs. Because these are the critical groups, their procedures will be described first. Procedures for Groups Cont, $\mathrm{Sac} \rightarrow \mathrm{nLi}$, and $\mathrm{Sac} \rightarrow \mathrm{fLi}$ will be described later.

Days 1-14: Preexposure-Throughout this period, each of 40 rats was visited for $2 \mathrm{~h}$ on odd days by a second rat in its home cage; each had $7 \mathrm{~h}$ of free access to unflavored water on even days while alone in its home cage. The pairing of rats always began when the rats had been deprived of water for $25-26 \mathrm{~h}$. In the case of Groups PP/Sac $\rightarrow$ nPP and PP/Sac $\rightarrow$ fPP $(n=10$ per group), each partner rat was injected ip with $2.5 \mathrm{ml}$ of $2 \%$ (weight/volume) lithium chloride solution just before being placed in the subject rat's home cage. In the case of Groups UP/Sac $\rightarrow$ nPP and UP/ $\mathrm{Sac} \rightarrow \mathrm{fPP}(\mathrm{n}=10$ per group), the partners were not poisoned. The rats were never paired with the same partner twice, and, indeed, they were exposed to still another partner during conditioning. This was to prevent the development of strong social relationships between specific rats. It also excluded a potential methodological problem: If the same partner were used throughout as much as feasible, there would be a necessary change in partners from preexposure to conditioning in Groups UP/Sac $\rightarrow \mathrm{fPP}$ and $\mathrm{PP} / \mathrm{Sac} \rightarrow$ nPP, but not in the other groups.

Days 15-17: Initiation of drinking regimen-All rats were limited to $10 \mathrm{~min}$ per day of free access to unflavored water.

Day 18: Conditioning-Each rat was given $10 \mathrm{~min}$ of free access to $.6 \%$ (weight/volume) sodium saccharin solution for $10 \mathrm{~min}$. If a rat failed to drink $2 \mathrm{~g}$ (as determined by weighing the bottles before and after the drinking period), about $2 \mathrm{ml}$ of the saccharin solution was passed over its tongue. This was necessary for four rats in Groups UP/Sac $\rightarrow$ nPP and UP/Sac $\rightarrow \mathrm{fPP}$, seven rats in Group PP/Sac $\rightarrow$ fPP, and three rats in Group PP/Sac $\rightarrow$ nPP. The high frequency of failures to drink is to be attributed mainly to the high concentration of saccharin flavoring. Just after the drinking period, a PP that had just been injected with $2.5 \mathrm{ml}$ of $2 \%$ $\mathrm{LiCl}$ solution $(50 \mathrm{mg}$ ) was placed in the cage of each animal for 
$2 \mathrm{~h}$ and then removed. In the case of Groups UP/Sac $\rightarrow \mathrm{nPP}$ and $\mathrm{PP} / \mathrm{Sac} \rightarrow \mathrm{nPP}$, the PP had never been subjected to $\mathrm{LiCl}$ sickness but had been used as a UP during the preexposure phase. In the case of Groups UP/Sac $\rightarrow \mathrm{fPP}$ and PP/Sac $\rightarrow \mathrm{fPP}$, the PPs had been poisoned and used as PPs during the preexposure phase. For half of the 10 rats in each group, the PP had consumed .6\% saccharin solution just before being injected; for the other half, the PP had not consumed any solution.

Days 19-25-On Days 20,22, and 24, the rats were allowed to drink the saccharin solution for $10 \mathrm{~min}$. On Days 19, 21, and 23, they were allowed to drink unflavored water for $10 \mathrm{~min}$. The consumption of unflavored water allowed rats with strong saccharin aversions to obtain adequate fluid.

For Group Cont, the defining characteristic was that these rats did not drink saccharin solution on the conditioning day. Instead, they drank unflavored water after exposure to the US. On Day 19, the day after the conditioning day, they drank .6\% saccharin solution for $10 \mathrm{~min}$ so that they would be just as familiar with its flavor as were the other rats. There were 28 rats in Group Cont. Eight rats were never directly exposed to $\mathrm{LiCl}$ sickness; for them the US used on the conditioning day was a PP. The remaining 20 rats functioned as PPs on the conditioning day. Hence, their US was $\mathrm{LiCl}$ sickness, which was experienced in the presence of an unpoisoned rat.

First we consider the eight rats for which the US was a PP. Each rat was subjected to a different procedure corresponding to the $2 \times 2 \times 2$ factorial combinations of different PPE procedures. Only on Days 18 and 19 did the procedures used differ from the PPE procedures. On Day 18, they received no fluid prior to introduction of the PP and $10 \mathrm{~min}$ of unflavored water $2 \mathrm{~h}$ after the PP was removed. On Day 19, they received $10 \mathrm{~min}$ of free access to $.6 \%$ saccharin solution instead of unflavored water. None of the eight rats failed to drink $2 \mathrm{~g}$.

The 20 rats in Group Cont that had been PPs on the conditioning day had been PPs or UPs during Days 1-14. Prior to the conditioning day, they were maintained on the same thirst schedule as all the other rats. On Day 18, the conditioning day, they did not drink any solution prior to being injected with $50 \mathrm{mg}$ of $\mathrm{LiCl}$, but were allowed to drink unflavored water for $10 \mathrm{~min}$, beginning $4 \mathrm{~h}$ after the $\mathrm{LiCl}$ injection. Two of the rats failed to drink $5 \mathrm{~g}$, probably due to lithium sickness, and were allowed to drink for an additional $10 \mathrm{~min}$. On Day 19, they drank .6\% saccharin solution instead of unflavored water. Only one of the 20 rats failed to drink $2 \mathrm{~g}$ and was passively exposed to $2 \mathrm{ml}$ of the solution. (The rats in Group Cont drank more saccharin solution during their first exposure to it than did the other rats. Perhaps this occurred because they drank the saccharin solution on the day after a disruption of their usual water drinking by the conditioning procedure and hence were thirstier.) From Day 20 to the end of the experiment, these rats, like the other rats in Group Cont, were treated in the same way as those subjected to PPE procedures.

For Groups $\mathrm{Sac} \rightarrow \mathrm{fl}, \mathrm{i}$ and $\mathrm{Sac} \rightarrow \mathrm{nLi}$, all rats were PPs on the conditioning day. They differed from the PPs in Group Cont in that they drank saccharin solution prior to induction of the sickness. The 14 rats in Group $\mathrm{Sac} \rightarrow \mathrm{fLi}$ had seven preexposures to lithium sickness while being used as PPs during Days 1-14, the preexposure phase. The 14 rats in Group Sac $\rightarrow$ nLi had been used as UPs during this period, and hence the Li sickness was novel to them on the day of conditioning. All these rats had 10 min to drink the $.6 \%$ saccharin solution just prior to being injected on Day 18. Seven rats in Group $\mathrm{Sac} \rightarrow \mathrm{fLi}$ and four rats in Group $\mathrm{Sac} \rightarrow \mathrm{nLi}$ failed to drink $2 \mathrm{~g}$ and were passively exposed to $2 \mathrm{ml}$ of the saccharin solution. In all unspecified respects, these groups were treated like those subjected to PPE procedures.

Saccharin preference ratios were obtained for Days 18, 21, 23, and 25 by dividing the amount of saccharin solution consumed on each such day by the sum of the amount consumed on that day and on the preceding water-drinking day. In the case of Group Cont, which did not drink saccharin solution on Day 18, the amount consumed on Day 19 was substituted and the amount of water consumed on Day 17 was used as the baseline. Statistical analysis of all results was for the first test day, Day 21 , only; data from later test days are reported, but they were not subjected to statistical test because weaker effects were expected after the first test day. The analysis of covariance with Day 18 as the covariate was used whenever appropriate since Archer and Sjöden (1979) had reported intersubject correlations that insured that such a method would increase sensitivity, an observation that has also been repeatedly made in our laboratory on a less formal basis. In the event of significant $(p<.05)$ inhomogeneity of regression, the same data were subjected to an ordinary ANOVA and the inhomogeneity was ignored if the same statistical decision was reached. We also used ordinary ANOVAs and t tests rather than a covariance analysis when Group Cont was involved because it did not drink saccharin solution on Day 18 in the same way as the other groups and therefore did not have similar preference ratios to be used as covariates.

The pooling of Group Cont over a variety of procedures was justified by an ANOVA comparing the eight subgroups. Those rats subjected to a PP as an unpaired US consisted of four subgroups of two rats each, factorially based on whether they had been preexposed to PPs or to UPs and whether the PPs used during conditioning were fPPs or nPPs. Those subjected to sickness as the unpaired US were in four subgroups of five rats each. These were based on the $2 \times 2$ combination of whether they had been PPs or UPs during the preexposure phase and whether the rats with which they were paired on the conditioning day had been preexposed to PPs or to UPs. The ANOVA showed little difference among these groups $[F(7,20)=.21]$. Similarly, Groups $\mathrm{Sac} \rightarrow \mathrm{fLi}$ and $\mathrm{Sac} \rightarrow \mathrm{nLi}$ were divided into subgroups on the basis of the type of rat with which they were paired on Day 18 . The results were similar over subgroups $[\mathrm{F}(3,9)<1.70$ in each case $]$.

\section{Results}

Figure 1 shows mean saccharin preference ratios for each group on each of the test days as well as on the conditioning day. First we will discuss the results for the 40 rats subjected to the PPE procedures. They drank saccharin solution prior to being subjected to the presence of a PP on the conditioning day. Recall that these rats were in a $2 \times 2 \times 2$ factorial design

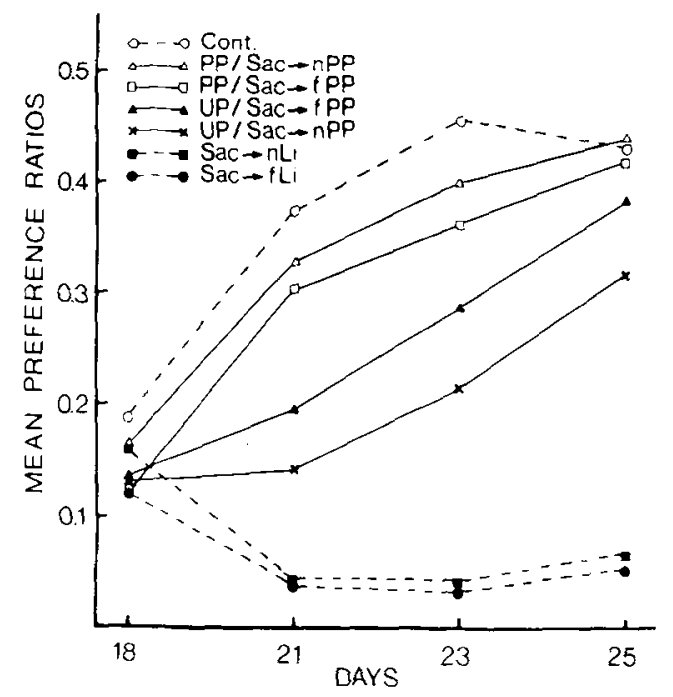

Figure 1. Saccharin preference ratios during training and testing for Experiment 1. The datum indicated for Day 18 was actually obtained on Day 19 in Group Cont. 
that had the following factors: (1) preexposure to a PP or control preexposure to a UP; (2) preexposure of their PPs to lithium sickness or not; (3) consumption by their PPs of saccharin solution prior to conditioning or not. The only significant result was the main effect of whether the rats had been preexposed to PPs or were subjected to the control procedure of preexposure to UPs $[F(1,31)=18.31, p<.001$, based on analysis of covariance]. This result shows that Groups PP/Sac $\rightarrow$ fPP and PP/Sac $\rightarrow$ nPP, taken together, had reliably higher preferences for saccharin on Day 21 than did Groups UP/Sac $\rightarrow$ fPP and $\mathrm{UP} / \mathrm{Sac} \rightarrow \mathrm{nPP}$. Thus, preexposure to PPs reduces the magnitude of the PPE. All other results, including interactions, yielded Fs $<1.0$, which shows that the magnitude of the PPE was not noticeably affected by whether the PP was familiar with sickness at the time of conditioning or had consumed saccharin solution prior to sickness.

Except for Groups PP/Sac $\rightarrow \mathrm{nPP}[\mathrm{t}(36)=1.52$, $p>.10]$ and $\mathrm{PP} / \mathrm{Sac} \rightarrow \mathrm{fPP}[\mathrm{t}(36)=2.26, \mathrm{p}<.05]$, for which the PPE had been weakened by preexposure to PPs, all groups subjected to the conditioning procedures exhibited reliable saccharin aversions relative to Group Cont. We do not believe that the apparent aversion in Group PP/Sac $\rightarrow$ fPP is statistically reliable, since the analysis of covariance eliminates its significance $[\mathrm{t}(35)=.71]$. Although, because the covariate baselines are different, the analysis of covariance is not applicable to comparisons of other groups with Group Cont by strict statistical criteria, the fact that such a comparison removes significance from this result suggests the possibility of an artifact. It may be recalled that the rats in Group Cont drank more saccharin solution on Day 19 than the other rats did on the conditioning day, and this increased consumption is capable of increasing preference due to greater attenuation of neophobia. The covariance analysis adjusts for this increased consumption. In all events, the groups preexposed to PPs exhibited weakened aversions, and the only issue in doubt is the extent of this weakening.

Groups $\mathrm{Sac} \rightarrow \mathrm{fLi}$ and $\mathrm{Sac} \rightarrow \mathrm{nLi}$ exhibited stronger aversions than the other groups (ps $<.001$, covariance) but did not differ from each other $[F(1,25)=$ .05 based on analysis of covariance].

\section{Discussion}

Preexposure to the presence of PPs reduced the capacity of PPs to produce flavor aversions. This US preexposure effect also characterizes taste aversion learning when toxicosis is the US. This occurrence of learned taste aversions with delays of up to $6 \mathrm{~h}$ between consumption of the saccharin solution and exposure to the PP (Coombes et al., 1980), indicates that exposure to the PP can be considered the same type of US as toxicosis. However, as Coombes et al.
(1980) also found, the PP is a weaker US in the sense that aversions in unpoisoned rats produced by PPs (solid curves in Figure 1) were far weaker than those produced by lithium poisoning itself (two bottom dashed curves of Figure 1).

One alternative to the theory that the PP acts like a US is that the taste aversion itself is somehow transferred from the PP to the unpoisoned rat. This alternative is excluded by the present confirmation of the finding of Coombes et al. (1980) that the PPE occurs regardless of whether the PPs have aversions to the test flavor.

Whether the PP was familiar with lithium sickness did not seem to affect its aversiveness as a US for the PPE, since there was no detectable difference between aversions produced by fPPs and those produced by nPPs. However, this negative result was not convincing because a positive effect might be expected to be small and methodologically difficult to obtain. The capacity of lithium to produce flavor aversions among the PPs themselves was not noticeably affected by whether they were preexposed to lithium (Groups $\mathrm{Sac} \rightarrow \mathrm{fLi}$ and $\mathrm{Sac} \rightarrow \mathrm{nLi}$ in Figure 1), and so it is not surprising that the capacity of PPs to produce flavor aversions was also not affected by whether they were preexposed to lithium. It is conceivable that the dose of lithium used in the present experiment was so high that a floor effect made it difficult to detect any effect of familiarity with the poison on the capacity of PPs to produce saccharin aversions. The US-preexposure effect can be difficult to detect. When Braveman (1975) showed that familiarity with lithium reduced its capacity to produce flavor aversions, his preexposure dose was $30 \%$ lower than that used here; he reduced this dose by another $50 \%$ during his conditioning phase. Similarly, Revusky and Taukulis (1975) used less than half of the present lithium dose in two conditioning sessions in which they tried to compare the capacity of familiar and unfamiliar contingent lithium sickness to produce flavor aversions. They did not obtain a noticeable difference in flavor preference between the familiarized and unfamiliarized groups until after about five extinction sessions. Given that the US preexposure effect can be so difficult to demonstrate in a straightforward paradigm, it is reasonable to suppose that it may be still more difficult to demonstrate its effect on the capacity of a PP to produce a flavor aversion.

\section{EXPERIMENT 2}

We wanted to find a lithium dose for Experiment 3 that would be low enough to exclude the floor effects conceivably responsible for the failure of Experiment 1 to demonstrate that the PPE is weakened if the PP is familiar with the poison. The doses used were $0,7.5,15.0,30.0$, and $60.0 \mathrm{mg}$ of $\mathrm{LiCl}$ per $200-\mathrm{g}$ 
rat, and all PPs drank saccharin solution prior to induction of the sickness on the conditioning day. Hence, we could compare the PPE with a learned aversion produced directly by poisoning at each of a number of doses. In traditional terms, the $0-\mathrm{mg}$ dose may be considered a CS-alone control, since placement together with an unpoisoned rat does not seem to be an effective US for the production of taste aversions (Coombes et al., 1980; Lavin et al., 1980).

\section{Method}

The 100 male Sprague-Dawley rats consisted of 10 groups of 10 rats each equated for body weight and treated in unspecified respects in the same way as in Experiment 1. Two days before the experiment, they ranged in weight from 188 to $207 \mathrm{~g}$. Their water was then removed, so that they were 2 days thirsty on Day 1 of the experiment. On Days $1-4$, they were allowed to drink unflavored water for $10 \mathrm{~min}$ per day. Conditioning occurred on Day 5 when all the rats were allowed $10 \mathrm{~min}$ of access to $.6 \%$ saccharin solution. Within a minute after the bottle was removed, four groups were injected ip with $7.5,15,30$, or $60 \mathrm{mg}$ of $\mathrm{LiCl}$ and a fifth group, the 0-mg group, was not injected. Within another minute, these groups were placed in the cages of five groups of unpoisoned rats for $2 \mathrm{~h}$ and then returned to their home cages. The unpoisoned rats had finished drinking their saccharin solution a minute or two before being presented with their PPs.

The number of rats that failed to drink $2 \mathrm{~g}$ of saccharin solution on the conditioning day was inexplicably less than in Experiment 1:6 of the total of 100 and no more than 1 in any group. As in Experiment 1, such rats were subjected to a passive exposure to the saccharin taste.

On Days 6 and 7, all rats were allowed 10 min access to unflavored water. On Day 8 , all rats were allowed 10 min access to the $.6 \%$ saccharin solution for a test of conditioning.

\section{Results and Discussion}

Figure 2 shows saccharin preferences on the test day for each group of rats. The upper curve shows

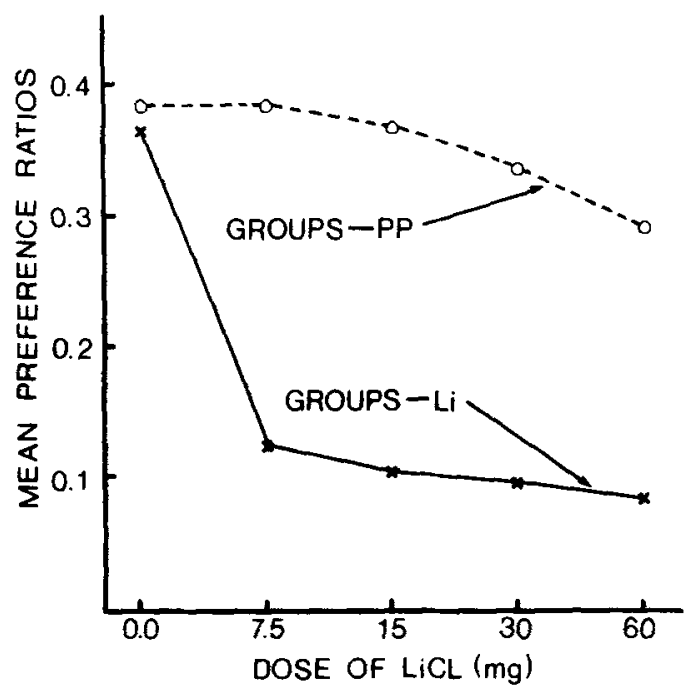

Figure 2. Saccharin preference ratios on the test day for rats subjected elither to various doses of lithium chloride after drinking seccharin solution (lower curve) or to the presence of a rat that bad been polsoned with some dose of hithium chloride (upper curve). results for groups subjected to a PP as the US, while the lower curve shows the results for the groups that were subjected to a lithium US. The PPE was inexplicably weaker than it had been in previous experiments. For instance, although the $60-\mathrm{mg} \mathrm{LiCl}$ dose was larger than the 50-mg dose used in Experiment 1, the PPE group exhibited a weaker aversion. In Experiment 1, Group UP/Sac $\rightarrow$ nPP exhibited a saccharin preference ratio of .14 on the first test while the 60 -mg LiCl PP group in Experiment 2 exhibited a corresponding saccharin preference ratio of .29; in both cases, the control level was .38. The effects obtained by Coombes et al. (1980) in their experiment with $.6 \%$ saccharin were even larger than those in Experiment 1. Nevertheless, the PPE was replicated in the present Experiment 2. We used the method of orthogonal polynomials to weight the results in the expected direction of an increasing effect with increases in the $\mathrm{LiCl}$ dose to which the PP was subjected (assuming equal spacing between each group); the 0 -mg dose was weighted -2 , the $7.5-\mathrm{mg}$ dose was weighted -1 , and so on. An analysis of covariance with Day 4 consumption, as the covariate yielded $\mathrm{p}<.05[\mathrm{~F}(1,44)=4.68]$; a similar analysis omitting the 0-mg (CS-alone) group also yielded a significant result $[F(1,35)=4.63, p<.05]$. Thus, the magnitude of the PPE was an increasing function of the $\mathrm{LiCl}$ dose to which the PP had been subjected, although even the $60-\mathrm{mg}$ group exhibited a nonsignificant aversion relative to the $0-\mathrm{mg}$ group $[\mathrm{t}(44)=1.90$, $p<.10$, based on the common error term and the adjusted means in the analysis of covariance for the groups subjected to PPE procedures].

There is nothing surprising about the data for the rats injected with $\mathrm{LiCl}$ after drinking the saccharin solution. The effect of different doses, although small, was significant $[F(1,35)=5.68, p<.05$, for the logarithmic trend omitting the 0 -mg group]. On the basis of the work of Revusky and Gorry (1973), we have no doubt that this trend would have become more obvious during an extinction phase. Of course, it was again confirmed that the aversions produced by $\mathrm{LiCl}$ itself were stronger than the PPE.

\section{EXPERIMENT 3}

Although a 30-mg dose of $\mathrm{LiCl}$ failed to produce a significant PPE in Experiment 2, we decided to use it in Experiment 3. The PPE in Experiment 2 seemed unusually weak, and we guessed that it might be stronger in Experiment 3. However, in an attempt to increase experimental sensitivity, we used a preexposure dose of $60 \mathrm{mg}$ of $\mathrm{LiCl}$, twice that used during conditioning (Braveman, 1975). Thus, Group Sac $\rightarrow$ fPP drank saccharin solution prior to being paired with a PP preexposed to lithium sickness, and Group Sac $\rightarrow$ nPP drank saccharin solution prior to being ex- 
posed to a PP not preexposed to lithium sickness. The primary question was whether Group Sac $\rightarrow$ fPP would exhibit higher saccharin preferences than Group Sac $\rightarrow$ nPP, thus indicating that an fPP, in its role as a US, produces weaker taste aversions than an nPP.

In addition, there were four other groups to allow answers to secondary questions. Group Cont simply drank saccharin solution without any experimentally manipulated aftereffect (CS-alone control) and hence supplied the saccharin preference level in the absence of a learned aversion. Group Sac $\rightarrow$ UP drank saccharin solution prior to being exposed to a UP. It was expected not to exhibit a saccharin aversion, in confirmation of earlier findings that a UP is not an effective US (Coombes et al., 1980; Lavin et al., 1980). To allow measurement of the aversion produced directly by the $\mathrm{LiCl}$, we permitted the PPs to drink saccharin solution prior to injection on the day of conditioning. Group Sac $\rightarrow \mathrm{fLi}$ was preexposed to the $\mathrm{LiCl}$ sickness, while Group Sac $\rightarrow \mathrm{nLi}$ was not.

\section{Method}

The 102 male Sprague-Dawley rats ranged in weight from 181 to $206 \mathrm{~g}$ on Day 0 , when they were deprived of water for the first time. In unspecified details, they were treated like rats in the earlier experiments. Until Day 15, all rats were maintained on cycles of $24 \mathrm{~h}$ of free access to unflavored water alternating with $24 \mathrm{~h}$ of deprivation; the $24 \mathrm{~h}$ of deprivation began during the afternoon of each day. On Days $1,3,5,7,9,11$, and 13, the 17 rats in Group $\mathrm{Sac} \rightarrow \mathrm{fLi}$ (destined to serve as fPPs) were injected with $3 \mathrm{ml}$ of $2 \%(\mathrm{w} / \mathrm{v})$ of $\mathrm{LiCl}$ solution $2.5 \mathrm{~h}$ before being given $24 \mathrm{~h}$ access to water. On Day 15, Group Sac $\rightarrow$ fLi was also subjected to a lithium injection but was not allowed water afterward. On Days 16, 17, and 18 , all rats were allowed 15 min per day of access to unflavored water.

On Day 19, the day of conditioning, all rats received $15 \mathrm{~min}$ of access to $.6 \%(\mathrm{w} / \mathrm{v})$ saccharin solution, but the treatments administered afterward varied. Right after drinking, each rat in Groups $\mathrm{Sac} \rightarrow \mathrm{fPP}$ and $\mathrm{Sac} \rightarrow \mathrm{nPP}(\mathrm{n}=17$ per group) had an $\mathrm{fPP}$ or an $\mathrm{nPP}$ placed in its cage for $2 \mathrm{~h}$. The fPPs and nPPs had just been injected with $1.5 \mathrm{ml}$ of $2 \%(\mathrm{w} / \mathrm{v}) \mathrm{LiCl}$ solution $(30 \mathrm{mg})$ after drinking saccharin solution and constituted Groups $\mathrm{Sac} \rightarrow \mathrm{fLi}$ and $\mathrm{Sac} \rightarrow \operatorname{nili}(\mathrm{n}=17$ per group). The 18 rats in Group Cont were not subjected to any experimental treatment after drinking saccharin solution. Finally, the 16 rats in Group $\mathrm{Sac} \rightarrow$ UP were subjected for $2 \mathrm{~h}$ to the presence of an unpoisoned rat. Half of the rats in Group Sac $\rightarrow$ UP were guests in the cages of the other half.

Only three rats (one each from Groups $\mathrm{Sac} \rightarrow \mathrm{fLi}, \mathrm{Sac} \rightarrow \mathrm{fPP}$, and $\mathrm{Sac} \rightarrow$ UP) failed to drink $2 \mathrm{~g}$ of saccharin solution on the day of conditioning, and they were subjected to a passive exposure to the saccharin taste.

On Days 20 and 21, all rats received 15 min access to unflavored water. Thereafter, saccharin solution was consumed on even days and unflavored water was consumed on odd days during the 15 min drinking period. The experiment ended after Day 26.

\section{Results}

As expected, Group Sac $\rightarrow \mathrm{fPP}$ exhibited weaker saccharin aversions than Group Sac $\rightarrow$ nPP, but the effect was very small. Figure 3 shows mean preference ratios for each group on each saccharin drinking day. An analysis of covariance for Day 22, with Day 19 as the covariate for Groups Cont, Sac $\rightarrow$ UP,

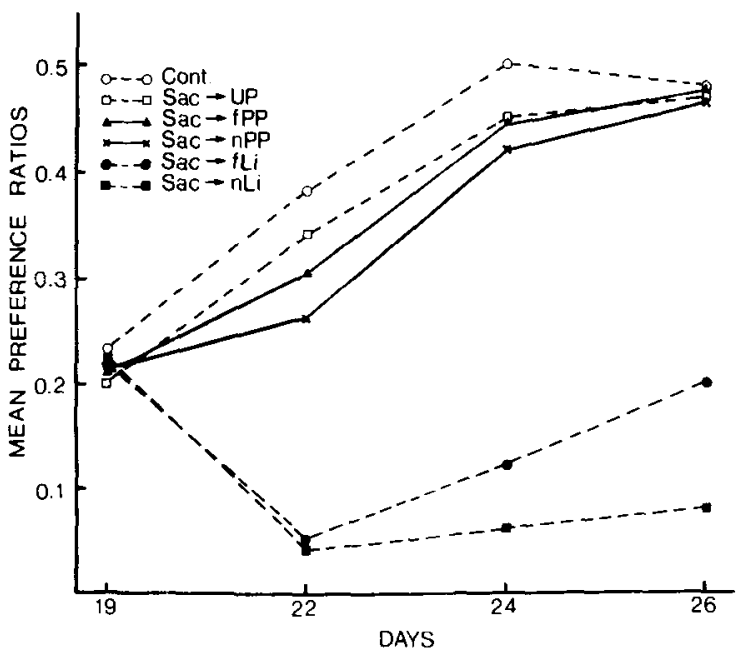

Figure 3. Saccharin preference ratios during training and testing for Experiment 3. Group Cont is a baseline control group; Group Sac $\rightarrow$ UP was exposed to unpoisoned rats after drinking saccharin solution. Groups Sac $\rightarrow$ fPP and Sac $\rightarrow$ nPP were exposed to poisoned rats, respectively familiar or unfamiliar with lithium poisoning, after drinking saccharin solution. Groups $S a c \rightarrow f L i$ and $S a c \rightarrow n L i$ were exposed, respectively, to familiar or unfamiliar lithium sickness after drinking saccharin solution.

$\mathrm{Sac} \rightarrow \mathrm{fPP}$, and $\mathrm{Sac} \rightarrow \mathrm{nPP}$, showed reliable differences among the groups $[F(3,63)=6.60, p<.001]$. In partial confirmation of the experimental hypothesis, Group Sac $\rightarrow$ nPP exhibited a saccharin preference somewhat lower than that of Group Sac $\rightarrow$ fPP, but at a marginal level of significance $[\mathrm{t}(63)=1.79$, $p<.10$, based on the analysis of covariance error term and the adjusted means]. Thus, preexposure of the PP to sickness may have weakened the PPE. There was also no reliable difference between Groups Cont and Sac $\rightarrow$ UP $[\mathrm{t}(63)=.63]$, confirming the earlier findings that exposure to a UP is not a US capable of producing a noticeable taste aversion. Group $\mathrm{Sac} \rightarrow \mathrm{nPP}$ exhibited clear-cut aversions relative to each of Groups Cont and Sac $\rightarrow$ UP [ts(63) > 3.35]. Group Sac $\rightarrow$ fPP exhibited a reliable saccharin aversion relative to Group Cont $[\mathrm{t}(63)=2.28, \mathrm{p}<.05]$, but not relative to Group $\mathrm{Sac} \rightarrow \mathrm{UP}[\mathrm{t}(63)=1.59]$. However, because there was no reliable difference between the latter two groups, it seemed reasonable to combine them, and, on this basis, there was good evidence that Group $\mathrm{Sac} \rightarrow \mathrm{fPP}$ exhibited a saccharin aversion $[t(64)=2.26, p<.05]$.

Groups $\mathrm{Sac} \rightarrow \mathrm{fLi}$ and $\mathrm{Sac} \rightarrow \mathrm{nLi}$ exhibited stronger flavor aversions than the other groups. Although Figure 3 shows only a tiny difference between these two groups on Day 22, it was significant according to the analysis of covariance $[F(1,31)=4.31, p<.05]$. The difference became larger on Days 24 and 26 $[F s(1,31)=9.43$ and 11.28 , respectively, with Day 19 as covariate; ps $<.005$ ] in a pattern similar to that reported by Revusky and Taukulis (1975). 


\section{Discussion}

Since the difference between Groups Sac $\rightarrow$ fLi and $\mathrm{Sac} \rightarrow \mathrm{nLi}$ was larger than the corresponding difference in Experiment 1, the strategy used to strengthen the effect of familiarization to the lithium in Experiment 3 was probably successful. We cannot be sure whether this strategy was responsible for the present success in demonstrating that preexposure of the PP to poisoning weakened its capacity to produce a PPE. In all cases, extraordinary steps were taken to increase experimental sensitivity, and if the usual methods had been used, this effect would probably not have been detected. We used 17 rats per group and the analysis of covariance to increase sensitivity, but still obtained only the small difference between Groups Sac $\rightarrow$ fPP and Sac $\rightarrow$ nPP shown in Figure 3 at a statistically marginal .10 level. Nevertheless, there is a good reason to believe that this small difference is real. As explained in the discussion of Experiment 1, it would not be reasonable to expect this effect to be very large. Preexposure to poisoning probably reduces the capacity of the PP to produce a PPE, because it gives the PP some tolerance to the poison so that it becomes less sick; presumably sickness produces whatever acts as a US for the unpoisoned partner, and when tolerance reduces this sickness, it weakens the PPE. It is often difficult to detect the effect of this tolerance on taste aversions directly produced by the poison; it was barely visible on the first day of testing, as is evident from the closeness of the Sac $\rightarrow \mathrm{nLi}$ and Sac $\rightarrow$ fLi data for Day 22 in Figure 3. Thus, it should be hardly surprising that the indirect effect of this tolerance in the comparison between Groups $\mathrm{Sac} \rightarrow \mathrm{nPP}$ and $\mathrm{Sac} \rightarrow \mathrm{fPP}$ should be very small. The small effect on the PPE is of interest, however, because it helps put the PPE into the same conceptual framework as aversions produced by direct administration of toxins.

\section{GENERAL DISCUSSION}

The general conclusion is that a PP is a US for flavor aversion learning and acts much like sickness itself, although it produces weaker aversions. Lavin et al. (1980) originally suggested this possibility, although they called the PPE a "transferred" flavor aversion. However, Coombes et al. (1980) and the present Experiment 1 showed that the PPE was not affected by whether the PP had a flavor aversion to transfer. An important similarity between the PP as a US and sickness itself as a US is that both can produce flavor aversions if presented as much as $6 \mathrm{~h}$ after consumption of a flavored solution (Coombes et al., 1980); the only other USs known to be effective over such long delays are toxins. Another similarity found in Experiment 1 was that the PPE was subject to the US habituation effect; that is, if the subject rat is familiar with PPs, the PP loses some of its capacity to produce flavor aversions, just as familiar toxicosis loses some of its capacity to produce flavor aversions (Braveman, 1975; Cappell \& LeBlanc, 1977; Revusky \& Taukulis, 1975). In Experiment 3, we demonstrated that if the PP is familiar with sickness, it loses some of its capacity to produce a flavor aversion. But this latter effect was very weak and difficult to detect.

We recognize the failure to suggest a direct mechanism for the PPE, or otherwise to theorize about it, as a weakness of this paper. We looked at the rats during exposure to the PP, but noticed no behavior that we thought might be responsible for the PPE. We have found in exploratory work, that if we combined urine and feces from a PP, we could obtain a flavor aversion by spreading them over the snout of an unpoisoned rat. This aversion was markedly weaker than the PPE. Urine alone or feces alone from a poisoned rat, or combined urine and feces from an unpoisoned rat, did not produce a noticeable aversion. Other potential USs that failed to produce flavor aversions were hair from a poisoned rat, a compartment in which a poisoned rat had recently been confined, and the presence of a rat recently sacrificed by means of carbon dioxide. This is about all we know, and we do not feel it is enough to justify theorizing.

\section{REFERENCES}

Archer, T., \& Suöden, P. O. Positive correlation between preand postconditioning saccharin intake in taste aversion learning. Animal Learning \& Behavior, 1979, 7, 144-148.

Braveman, N. S. Formation of taste aversions in rats following prior exposure to sickness. Learning and Motivation, 1975, 6, 182-194.

Cappell, H. D., \& LeBlanc, A. E. Gustatory avoidance conditioning by drugs of abuse: Relationship to general issues in research on drug dependence. In N. W. Milgram, L. Krames, \& T. M. Alloway (Eds.), Food aversion learning. New York: Plenum Press, 1977.

Carroll, M., Dinc, H., Levy, C., \& Smith, J. C. Demonstrations of neophobia and enhanced neophobia in the albino rat. Journal of Comparative and Physiological Psychology, 1975, 5, 219-220.

Coombes, S., Revusky, S., \& LetT, B. T. Long-delay taste aversion learning in an unpoisoned rat: Exposure to a poisoned rat as the US. Learning and Motivation, 1980, 11, 256-266.

DomJan, M. Attenuation and enhancement of neophobia for edible substances. In L. M. Barker, M. R. Best, \& M. Domjan (Eds.), Learning mechanisms in food selection. Waco, Tex: Baylor University Press, 1977.

Gamzu, E. The multifaceted nature of taste-aversion inducing agents: Is there a single common factor? In L. M. Barker, M. R. Best, \& M. Domjan (Eds.), Learning mechanisms in food selection. Waco, Tex: Baylor University Press, 1977.

Lavin, M. J., Freise, B., \& Coombes, S. Transferred flavor aversions in adult rats. Behavioral and Neural Biology, 1980, 28, 15-33.

Mis, F. W., \& Moone, J. W. Effect of preacquisition UCS exposure on classical conditioning of the rabbit's nictitating membrane response. Learning and Motivation, 1973, 4, 108-114. 
Rescorla, R. A. Effect of US habituation following conditioning. Journal of Comparative and Physiological Psychology, 1973, 82, 137-143.

Revusky, S. Reply to Mitchell. Animal Learning \& Behavior, 1978, 6, 119-120.

REvusky, S. More about appropriate controls for taste aversion learning: A reply to Riley. Animal Learning \& Behavior, 1979, 7, 562-563.

Revusky, S. H., \& Gorky, T. Flavor aversions produced by contingent drug injection: Relative effectiveness of apomorphine, emetine, and lithium. Behaviour Research and Therapy, 1973, 11, 403-409.

Revusky, S., Parker, L. A., Coombes, J., \& Coombes, S. Rat data which suggest alcoholic beverages should be swallowed during chemical aversion therapy, not just tasted. Behaviour Research and Therapy, 1976, 14, 189-194.

Revusky, S., \& TaukULis, H. K. Effects of alcohol and lithium habituation on the development of alcohol aversions through contingent lithium injection. Behaviour Research and Therapy, 1975, 13, 163-166.

Revusky, S., Taukulis, H. K., Parker, L. A., \& Coombes, S. Chemical aversion therapy: Rat data suggest it may be countertherapeutic to pair an addictive drug state with sickness. Behaviour Research and Therapy, 1979, 17, 177-188.

(Manuscript received January 6, 1981; revision accepted for publication August 26, 1981.) 